\title{
FELICIDADE: A ESPIRITUALIDADE COMO POSSÍVEL CAMINHO
}

\author{
Happiness: Spirituality as a Possible Way
}

Anna Luiza Da Silva Miranda Varella Souza ${ }^{1}$ Andrés Eduardo Aguirre Antúnez ${ }^{2}$

\section{RESUMO}

O presente artigo destaca possíveis articulações existentes na literatura sobre felicidade e espiritualidade. As literaturas psiquiátrica, psicológica e filosófica norteiam a existência humana e, segundo estudos recentes, se dá por meio da manutenção de relações positivas e profundas com os outros, conquista da autonomia, domínio do ambiente, crescimento pessoal, propósito na vida e autoaceitação — aspectos fortemente relacionados com o desenvolvimento da espiritualidade. Estudos científicos mostram que pessoas mais espiritualizadas apresentam relações mais fortes e positivas, autonomia, crescimento pessoal, sentimento de pertencimento, sentido na vida e, portanto, aumento da sensação de bem-estar. Assim, as pessoas podem atingir a felicidade de viver e ter acesso ao encontro com o próprio poder por meio do reconhecimento da espiritualidade e da transcendência humana.

Palavras-chave: Felicidade; Espiritualidade; Transcendência; Saúde Mental.

\begin{abstract}
This article highlights possible articulations existing in the literature on happiness and spirituality. Psychiatric, psychological and philosophical literature guides human existence and, according to recent studies, it occurs through the maintenance of positive and profound relationships with others, the achievement of autonomy, control of the environment, personal growth, purpose in life and self-acceptance. Which is strongly related to the development of spirituality. Scientific studies show that more spiritualized people have stronger and more positive relationships, autonomy, personal growth, feeling of belonging, meaning in life and, therefore, a feeling of greater wellbeing. Thus, recognizing spirituality and human transcendence can give people, sick or not, their own power and happiness to live.
\end{abstract}

Keywords: Happiness; Spirituality; Transcendence; Mental Health.

\footnotetext{
${ }^{1}$ Mestranda do Programa de Pós-graduação em Psicologia Clínica, Instituto de Psicologia da Universidade de São Paulo.

https://orcid.org/0000-0002-6926-3381 http://lattes.cnpq.br/9467581537770283

${ }^{2}$ Professor Associado do Departamento de Psicologia Clínica, Instituto de Psicologia; Professor subsidiário do Instituto de Psiquiatria, Faculdade de Medicina, Coordenador do Escritório de Saúde Mental da Pró-Reitoria de Graduação da Universidade de São Paulo.

https://orcid.org/0000-0001-5317-4459

http://lattes.cnpq.br/7711423427014371
} 


\section{Introdução}

O desenvolvimento da espiritualidade e, consequentemente, da sensação de bem-estar — resultado daquela — , se destaca como um possível recurso para melhorar a vida da população, tanto como uma forma de aprimorar a qualidade de vida, quanto para prevenir e tratar transtornos de humor. Segundo dados publicados pela Organização Mundial da Saúde (2017), o Brasil é o país com mais casos de ansiedade no mundo, correspondendo a 9,3\% da população, enquanto a média mundial é de 5,6\%. O país encontra-se acima da média também em casos de depressão — enquanto 4,4 \% da população mundial sofre com esse transtorno, no Brasil são afetados 5,8\% dos seus habitantes.

Portanto, faz-se necessário, diante da urgência dessa demanda, estudar e pensar formas que possam auxiliar na diminuição dessas taxas ou na prevenção de números ainda piores. Para isso, este artigo pretende compreender as interlocuções existentes entre felicidade e espiritualidade, visto que podem ser um possível recurso para aprimorar a qualidade de vida da população, conforme ressaltado anteriormente.

\section{FELICIDADE}

A busca pela felicidade norteia a existência humana. Por trás de parte de nossos atos, enquanto seres humanos, encontra-se o mesmo princípio, a mesma crença de que nossas escolhas nos tornarão ao menos um pouco mais felizes.

Entretanto, não compreender o que é felicidade e o que nos torna mais felizes gera uma série de enganos e desventuras ao longo da trajetória da vida. Lyubomirsky $(2008,2013)$ busca desmistificar a felicidade como algo a ser alcançado, fruto do sucesso ou de aquisições futuras. Para a autora, felicidade consiste em um treino diário que abarca uma série de conceitos tais como cultivar pensamentos positivos, reler a própria história e à do mundo sob outra perspectiva, bem como ter relacionamentos sociais profundos ao longo da vida.

Pesquisadores contemporâneos como Seligman (2009) e Lyubomirsky (2008, 2013) propõem que conhecer as principais forças de caráter presentes em nós e aprender a usá-las — além 
de autoconhecimento — também traz resultados positivos em todas as esferas, como o aumento da qualidade de vida e elevação do nível de bem-estar. Os autores supracitados desconstroem a ideia de que a felicidade está restrita ao sucesso profissional e à riqueza material. Além disso, destacam a importância do momento presente, da gratidão, da gentileza, da conexão social, do autoconhecimento, de experienciar o mundo, ter suas necessidades básicas atendidas e, então, trabalhar por ver um sentido naquilo que se faz. Curiosamente, ambos ressaltam como medidas práticas os benefícios da meditação, do exercício voluntário da gratidão, de colocar o corpo em movimento por meio de atividades físicas e, no que diz respeito ao bem-estar, da interferência do ambiente no qual esteja inserido.

O pesquisador visitante da Universidade de Stanford e tradutor dos livros do Dalai Lama, Jinpa (2016) - que é ex-monge - sugere que o conceito de felicidade está calcado no autoconhecimento e na autoaceitação, manutenção de relações profundas e de autonomia, domínio do ambiente em que esteja inserido, e, a partir deste, a busca de sentido para a vida. Logo, devese encarar os acontecimentos como uma oportunidade de crescimento no âmbito pessoal.

O autor ainda sublinha a compaixão e a busca pela felicidade como pontos que nos unem enquanto seres humanos. A partir da humanidade que temos em comum, surge como uma oportunidade de estabelecer uma conexão profunda com todos os seres (JINPA, 2016).

A própria escala de medição de nível de bem-estar psicológico — adaptada e validada no Brasil por Machado, Bandeira e Pawlowski (2013) — usa seis dimensões que estão positivamente associadas à satisfação com a vida para a mensuração, tais como: relação positiva com os outros; autonomia; domínio do ambiente; crescimento pessoal; propósito na vida; e autoaceitação.

Chama a atenção que as descrições citadas anteriormente se assemelham à busca pela espiritualidade. Muitas das formas propostas de encontrar a felicidade se entrelaçam com a literatura sobre essa temática.

\section{ESPIRITUALIDADE}

O conceito de espiritualidade abordado aqui se diferencia da ideia de religiosidade, visto que a segunda, por mais que busque aproximar o individuo da espiritualidade e do sagrado, envolve 
uma instituição, crenças e rituais pré-estabelecidos e compartilhados por um grupo que é atravessado por questões culturais e sociais especificas. Compreende-se que parte da população se conecte com a sua espiritualidade por via da religião. Entretanto, o conceito de espiritualidade apresentado neste artigo almeja alcançar outras formas também possíveis de vivenciá-la, de formas subjetivas. Participantes de uma pesquisa realizada em um hospital de Juiz de Fora afirmam que há a possibilidade de ser espiritualizado sem ser religioso, assim como se pode atribuir maior relevância às vivências intrínsecas em detrimento das institucionais (CURCIO; MOREIRAALMEIDA, 2019).

Espiritualidade, segundo Lotufo Neto (1997, p.10), “trata-se da busca humana por uma vida satisfatória e com sentido, descobrindo a natureza essencial de si mesmo e seu relacionamento com o universo".

O autor supracitado acrescenta ainda ser “o processo pelo qual os indivíduos reconhecem a importância de orientar suas vidas a algo não material que está além e é maior do que eles próprios” (LOTUFO NETO, 1997, p.10).

Para Gomez e Fisher (2013), ter saúde e bem-estar espiritual é a união de relacionamentos profundos e saudáveis, por vezes transcendentes, consigo mesmo (nível pessoal), por meio do encontro com seus valores, significados, finalidade, e o desenvolvimento de um sentido para a vida; com a natureza (nível ambiental) — através do cuidado com o meio ambiente —, reconhecimento da fonte da vida, contemplação e sentimento de unidade; com Deus ou Ser Superior (nível transcendente) que envolve a fé, contemplação, percepção da presença desse outro na sua vida; e com o outro (nível comunitário), pelo relacionamento interpessoal, moralidade, cultura e exercício da religião.

Ainda sobre a relação com o outro, é importante destacar a importância da empatia nesse contato, considerada como essencial para a compreensão da outra pessoa e que, através dessa abertura, se torna possível enxergar o outro como um semelhante. Sendo uma porta de entrada à esfera espiritual, pelo nível interpessoal. Afinal, como ressalta Edith Stein, filósofa que aborda com excelencia essa temática, em seus escritos sobre a empatia, destaca ser inviável alcançar tal compreensão espiritual, que vai além do humano, focado apenas em si próprio (JOSGRILBERG, 
2013). A abertura genuína para o outro é parte significativa do processo e pode ser desenvolvida por meio do exercicio da empatia.

Segundo Vaillant (2010), a união com outros seres nos fornece um conjunto de emoções positivas, tais como amor, alegria, perdão, esperança, compaixão, fé, reverência e gratidão. E permite uma experiencia particular e subjetiva com o divino, seja lá como este é representado para o indivíduo em questão.

A trajetória de vida da filósofa alemã Edith Stein pode ser apontado como um exemplo da vivência da espiritualidade e a busca pessoal por respostas existenciais, que ocorreu em paralelo com o seu singular processo de individuação. Frente à consciencia que tinha de si, Stein esteve comprometida com a busca por um sentido na vida, pela verdade e pela empatia em relação ao outro (ANDREATA, 2019).

\section{INTERLOCUÇÕES}

Estudos que relacionam as duas temáticas apresentam que pessoas mais espiritualizadas apresentam melhor saúde física e mental e possuem melhor resposta a eventos estressantes. Quanto maior a espiritualidade, maior o sentimento de bem-estar e o nível de felicidade (PANZINI et al., 2007). Um estudo com crianças e adolescentes de Porto Alegre apontou uma relação entre altos níveis de espiritualidade e/ou religiosidade com níveis elevados de felicidade e maior saúde mental (VALDIVIA, 2017). Já outra pesquisa, com idosos em uma casa de repouso, mostrou resultados semelhantes - quanto maior a espiritualidade e religiosidade, maior a qualidade de vida (CARLOS, 2017). O mesmo foi encontrado em pacientes com insuficiência renal crônica em hemodiálise, em um hospital de Juiz de Fora (TOSTES, 2018). Moreira-Almeida e Lotufo Neto (2006), em uma revisão bibliográfica, expõem que, além de altos níveis de espiritualidade estarem associados ao bem-estar, há características relacionadas a melhores índices de saúde mental, tais como menor incidência de pensamentos suicidas, depressão e abuso de álcool e drogas .

Pessoas mais espiritualizadas apresentam relações mais fortes e positivas, propósito na vida, autonomia, crescimento pessoal, pertencimento, sentido na vida e, portanto, sentimento de maior bem-estar (GREENFIELD; VAILLANT ; MARK, 2009). 
A ausência da sensação de bem-estar e de sentido na vida são traços marcantes daqueles que sofrem de ansiedade e depressão. Por vezes, apresentam também baixa sensação de pertencimento e, em casos graves, perdem sua autonomia. A lembrança dos aspectos espirituais presentes também nestes, mas talvez adormecidos, pode ser uma forma de resgatar ou aprimorar aspectos até então adoecidos.

Gonçalves et al. (2015) demonstram que intervenções com meditação e assistência pastoral refletem em diminuição da ansiedade e melhora no quadro depressivo.

Mosqueiro, Rezende Pinto e Moreira-Almeida (2020) também ressaltam que a espiritualidade se apresenta como uma forma eficiente de intervenção e prevenção de transtornos de humor. Portanto, surgem como promissoras as psicoterapias que abordem a espiritualidade de forma integrada. Nesse âmbito, faz-se necessário repensar a clínica e pensar na forma de abordar tal aspecto constituinte do ser humano.

Ales Bello (2019), ao estudar a vida e obra de Edith Stein, mostra que a unidade do ser humano consiste na união de sua dimensão corporal, psíquica e espiritual. Corresponde a dimensão espiritual a liberdade humana, que compreende desde o ato de avaliar uma situação e tomar uma decisão, até o aspecto mais profundo de nível teológico — o encontro com o sagrado. Ambas as dimensões são independentes e complementares ao mesmo tempo. Portanto, é clara a importancia de que cada pessoa, à sua maneira, encontre formas de desenvolver e viver sua espiritualidade, sendo essencial para quem almeja certa proximidade com a completude.

Importante destacar que as pessoas consideradas mais espiritualizadas não o são necessariamente, mas acabam tornando-se. É possivel desenvolver a espiritualidade. A capacidade humana para as emoções positivas nos torna aptos para a espiritualidade. Caminhar por essa via para alcançá-la é o melhor e mais seguro método para vivenciá-la. Todos nós temos a capacidade de experimentar momentos de conexão intensa com o outro e de reverência profunda ao longo da vida. Mesmo que seja necessário um estímulo externo para tal, seja o contato com a natureza, com a arte, com o humano, dentre tantas formas (VAILLANT, 2010). Portanto, este é um caminho inclusivo, um convite em aberto, possível para todos que almejarem.

\section{CONSIDERAÇÕES FINAIS}

REVIST A RELEGENSTHRÉSKEI A-2021-UFPR 
Estudos que relacionam as duas temáticas fornecem fortes evidências do quanto o desenvolvimento da espiritualidade pode elevar o nível de bem-estar, assim como auxiliar no tratamento e prevenção de transtornos ansiosos e depressivos. Sendo o desenvolvimento e a vivência da espiritualidade uma possível fonte de felicidade.

Torna-se evidente que, diferentemente da literatura sobre felicidade, o que é visto sobre espiritualidade não fornece maneiras práticas de prová-la em nossas vidas. Fica subentendido que cada um possui um caminho, um modo subjetivo de vivê-la. Entretanto, surge o questionamento se não há semelhanças nesses caminhos, que, caso sejam mais bem explanados, podem ser aderidos por outros.

Moreira-Almeida, Lotufo Neto e Koenig (2006) ressaltam que ainda são necessários estudos que apontem os mediadores desta associação e formas de aplicação deste conhecimento na prática. Portanto, é de bem comum investigar meios de experienciar a espiritualidade, a fim de fornecer à população mais uma forma de aprimorar sua qualidade de vida.

Sobre o fundo dos conhecimentos da medicina, sempre novos e em contínuo progresso

[...] algo de diferente está em jogo em tudo o que supõe uma relação intersubjetiva concreta que jamais é redutível ao saber objetivo, por mais elaborado que ele seja. O olhar médico é um olhar transcendental: através do dado científico, ele visa o que chamamos uma vida fenomenológica sempre singular (HENRY, 2014, p. 44).

O exame de uma radiologia procura ver, através dela, o sofrimento que trata de afastar ou tornar suportável.

Com mais forte razão no domínio da psiquiatria, é sempre esta vida dos indivíduos que define o verdadeiro tema da investigação, da teoria e da terapia. A interdisciplinaridade já não é aqui um piedoso voto, é o trabalho e o pão quotidiano daqueles que, psiquiatras e filósofos, uma mesma finalidade reúne; devolver uma vida doente, talvez doente de uma doença mortal, ao seu poder e felicidade de viver" (HENRY, 2014, p.44).

A apreciação do papel da espiritualidade e da experiência religiosa na vida de uma pessoa apresenta conseqüências relevantes para a prática psicoterapêutica, pois a avaliação da qualidade destas experiências amplifica a compreensão sobre os significados das queixas e dificuldades 
psicológicas. É, assim, um importante indicador de como a pessoa se apresenta no mundo, como concebe o divino e o sagrado (GENARO JUNIOR, 2011).

Portanto, é imprescindível investigarmos a espiritualidade do ser humano e sua relação na busca da felicidade, e não deixá-la distanciada ou afastada, pois, assim, corre-se o risco de uma compreensão e intervenção clínica que não integra nem acolhe a complexidade humana. A dimensão espiritual tende para a busca da felicidade como fator contemplativo de equilíbrio, harmonia e amadurecimento.

\section{REFERÊNCIAS}

ALES BELLO, A. O Sentido do Humano: entre fenomenologia, psicologia e psicopatologia. São Paulo: Paulus, 2019.

ANDREATA, Ocir de Paula. Individuação e experiência religiosa em Edith Stein. Relegens Thréskeia, Curitiba, v. 7, n. 2, p. 152-163, 2019.

CARLOS, Renata Gisela Pereira Ferreira. Influência da Espiritualidade no Idoso. 2017. 56 p. Dissertação (Mestrado em Psicologia Clínica) - Especialização em Psicoterapia e Psicologia Clínica, Instituto Superior Miguel Torga, Coimbra - Portugal, 2017.

CURCIO, C. S. S.; MOREIRA-ALMEIDA, A. Investigação dos conceitos de religiosidade e espiritualidade em amostra clínica e não clínica em contexto brasileiro: uma análise qualitativa. Interação em Psicologia, Curitiba, v. 23, n. 2, 2019.

GENARO JUNIOR, F. Psicologia clínica e espiritualidade/ religiosidade: interlocução relevante para a prática clínica contemporânea. Psicologia Revista, São Paulo, v. 20, n. 1, p. 29-41, 2011.

GOMEZ, R.; FISHER, J. W. Domains of spiritual well-being and development and validation of the Spiritual Well-eing Questionnaire. Personality and Individual Differences, n. 35, p. 19751991, 2013.

GONÇALVES, J. P. B.; LUCCHETTI, G.; MENEZES, P. R.; VALLADA, H. Religious and spiritual interventions in mental health care: A systematic review and meta-analysis of randomized controlled clinical trials. Psychological Medicine, v. 45, n. 14, p. 2937-2949, 2015. https://doi.org/10.1017/s0033291715001166.

GREENFIELD, E. A.; VAILLANT, G. E.; MARKS, N. F. Do formal religious participation and spiritual perceptions have independent linkages with diverse dimensions of psychological wellbeing? Journal of Health and Social Behavior, v. 50, n. 2, p. 196-212, 2009. 
HENRY, Michel. Sofrimento e Vida. In: ANTÚNEZ, A. E. A.; MARTINS, F.; FERREIRA, M. V. (Orgs.). Fenomenologia da vida de Michel Henry - interlocuções entre Filosofia e Psicologia. São Paulo: Editora Escuta, 2014. pp. 33-44.

JINPA, Thupten. Um coração sem medo. Rio de Janeiro: Sextante, 2016.

JOSGRILBERG, Rui. Vivência filosófica e espiritualidade cristã em Edith Stein. Revista Notandum, Cemoroc-Feusp/IJI-Universidade do Porto, Porto, Portugal, n. 33, p. 13-22, 2013.

LOTUFO NETO F. Psiquiatria e Religião - A Prevalência de Transtornos Mentais entre Ministros Religiosos. 1997. 375 p. Tese (Livre-Docência) - Faculdade de Medicina, Departamento de Psiquiatria, Universidade de São Paulo, São Paulo, 1997.

LYUBOMIRSKY, Sonja. A Ciência da Felicidade. 1. ed. São Paulo: Elsevier, 2008.

LYUBOMIRSKY, Sonja. Os mitos da felicidade. Rio de Janeiro: Odisseia Editorial, 2013.

MACHADO, W; BANDEIRA, D; PALOWSKI, J. Validação da Psychological Well-Being Scale em uma amostra de estudantes universitários. Revista Avaliação Psicológica, Campinas, v. 12, n. 2, p. 263-272, 2013.

MOREIRA-ALMEIDA, Alexander; LOTUFO NETO, Francisco; KOENIG, Harold G. Religiousness and mental health: a review. Revista Brasileira de Psiquiatria, São Paulo, v. 28, n. 3, p. 242-250, 2006 Sept. Disponível em: $<$ http://www.scielo.br/scielo.php?script=sci_arttext\&pid=S151644462006000300018\&lng=en\&nrm=iso>. Acesso em: 23 mai. 2020. https://doi.org/10.1590/S1516-44462006005000006.

MOSQUEIRO, B. P.; REZENDE PINTO, A.; MOREIRA-ALMEIDA, A. Spirituality, Religion and Mood Disorders. In: ROSMARIN, H. D; HAROLD, G.; KOENIG, G. H. (Eds.). Handbook of Spirituality, Religion, and Mental Health. $2^{\text {nd }}$. ed. London, UK: Academic Press, 2020. p. 125.

ORGANIZAÇÃO MUNDIAL DE SAÚDE — OMS. Depression and Other Common Mental Disorders - Global Health Estimates. OMS, Genebra, 2017. Disponível em: <http://apps.who.int/iris/bitstream/handle/10665/254610/WHO-MSD-MER-2017.2-

eng.pdf;jsessionid=342078279FBE7908AD69E88C4537501B? sequence=1>. Acesso em: 10 mai. 2020 .

PANZINI, Raquel Gehrke et al. Qualidade de vida e espiritualidade. Revista de Psiquiatria Clínica, São Paulo, v. 34, supl. 1, p. 105-115, 2007 . Disponível em: $<$ http://www.scielo.br/scielo.php?script=sci_arttext\&pid=S0101- 
$60832007000700014 \& \operatorname{lng}=\mathrm{en} \& \mathrm{nrm}=\mathrm{iso}>$. Acesso em: 22 mai. 2020. https://doi.org/10.1590/S0101-60832007000700014.

SELIGMAN, Martin E. P. Felicidade Autêntica. São Paulo: Objetiva, 2009.

TOSTES, Janaína Siqueira Rodrigues Martins. Associação entre religiosidade e felicidade em pacientes renais crônico em hemodiálise: estudo longitudinal. 2018. 115 f. Tese (Doutorado em Saúde) - Faculdade de Medicina, Programa de Pós-Graduação em Saúde Brasileira, Universidade Federal de Juiz de Fora, Juiz de Fora, 2018.

VAILLANT, George E. Fé: Evidências científicas. 1. ed. Barueri, SP: Manole, 2010.

VALDIVIA, Lucianne. Associação entre felicidade e espiritualidade em crianças e adolescentes saudáveis em escolas de Porto Alegre. 2017. 114 f. Dissertação (Mestrado em Psiquiatria e Ciências Comportamentais) - Faculdade de Medicina, Programa de Pós-Graduação em Psiquiatria e Ciências Comportamentais, Universidade Federal do Rio Grande do Sul,. Porto Alegre, 2017. 